\title{
Nutritional Epigenetics
}

\author{
L. Preston Mercer* \\ Department of Community and Family Health, University of South Florida, USA
}

Received: November 12, 2013; Accepted: December 10, 2013; Published: December 23, 2013

*Corresponding authors: L. Preston Mercer, Department of Community and Family Health, University of South Florida, USA, Tel: 813-974-9981; Fax: 813-974-8889; E-mail: pmercer@usf.edu

\begin{abstract}
Questions concerning the fundamental effects of nutrition on gene function are now being elucidated as the human genome project has been completed. Nutritional genomics seeks to expand the use of foods to achieve human genetic potential, while reducing the risk of diseases. As issues such as nutrigenomics (dietary influence on gene function) and nutrigenetics (genomic reaction to diet) are unraveled, thepotential for personalized nutrition becomes attainable. It has been stated that "genomics is to the 21 st century what infectious disease was to the 20th century". The nucleotide sequence of DNA was once seen as the only mechanism by which genetic information could be transmitted between generations. Phenotypic variation resulted from recombination and, occasionally, genetic mutation. This widely accepted concept is now undergoing modification as evidence builds to support the idea that reversible, heritable changes in gene function - termed "epigenetics"- can occur without a change in the sequence of nuclear DNA (i.e., non-Mendelian inheritance). The word epigenetics is of Greek origin and literallymeans over and above (epi) the genome. The terminology"same genome, different epigenome" has been demonstrated in several experiments. As research and understanding advances, dietary advice based on the human genome will become more prevalent and new pharmacological interventions may be developed.
\end{abstract}

Keywords: Genetics; Nutrition; Epigenetics; Public health, Obesity; Global

Since Hippocrates' axiom in $370 \mathrm{BC}$, "Thy food shall be thy medicine", the relationship between food, health and quality of life has been a concern of the scientific health care community. Of course, the boundary between medical (therapeutic) and normal nutrition changes regularly, with many opinions articulated in both the scientific community and the general population. Opinions vary from evidence-based medicine to anecdotal outcomes based on personal experience. When teaching nutrition, one knows that the presentations and responses can be heated, and a corollary lecture on the pitfalls of inductive logic is not only useful, but often necessary.

To bring clarity to the nutrition and health issue, questions concerning the fundamental effects of nutrition on gene function are now being elucidated as the human genome project has been completed [1]. Nutritional genomics ("omics" - the study of) seeks to expand the use of foods (or food components) to achieve human genetic potential while reducing the risk of diseases. As issues such as nutrigenomics (dietary influence on gene function) and nutrigenetics (genomic reaction to diet) are unraveled, the potential for personalized nutrition becomes attainable. In fact, it has been stated that "genomics is to the $21^{\text {st }}$ century what infectious disease was to the 20th century". [2]

However, implementation of nutritional genomics brings into focus one of the limiting foundations of scientific inquiry: the reductionist approach of a single perturbation of a system followed by observation and conclusions. This ignores the real concept of systems biology, macroscopic statistical analyses and the complexity of interactions producing "big data".[3] With humans having individual genomic profiles, cultural norms, socio-economic potential, religious food guidelines, etc., the goal of predictive and or mathematical rigor, while desirable, seems nearly unachievable. Data mining and complex algorithms will be used extensively.

New findings make the problem even more complex. The nucleotide sequence of DNA was once seen as the only mechanism by which genetic information could be transmitted between generations. Phenotypic variation resulted from recombination and, occasionally, genetic mutation. This widely accepted concept is now undergoing modification as evidence builds to support the idea that reversible, heritable changes in gene function - termed "epigenetics"- can occur without a change in the sequence of nuclear DNA (i.e., non-Mendelian inheritance). The word epigenetics is of Greek origin and literally means over and above (epi) the genome. Closer examination has revealed a multifaceted genetic regulatory system involving DNA methylation, histone modification, nucleosome location and/ or noncoding RNA. Genes can be viewed as "switches" which can be turned "on" or "off" or "up" or "down", independently of nucleotide sequence (also called genomic imprinting), affecting gene expression by chemically modifying DNA and/or altering the chromatin structure. Exposure to specific nutrients or toxins, certain behavioral patterns and other types of environmental factors can influence gene expression, without altering the genetic code at all. Surprisingly, such switches seem to be transmitted to subsequent generations. For example, "tags" (such as DNA methylation) were thought to be removed from the chromosome when the zygote was formed, but now it is believed that some of these tags remain, thereby passing the parents' experiences on 
to offspring and even succeeding generations. The terminology "same genome, different epigenome" has been demonstrated in several experiments, including the well-known work of Water land and Jirtle using mice with the Agouti gene where the Agouti gene is "turned off" by methylation. [4] Another example is the extended life span of yeast through caloric restriction, which silences certain genes. [5,6] Environmentally-induced epigenetic transgenerational inheritance of phenotype and disease is being elucidated and confirmed. [7]

One possible application of these findings understands the observed, world-wide increase in obesity, even in the very young. Since 1978, the number of overweight (BMI above the $85^{\text {th }}$ percentile) children has tripled. Moreover, 20092010 U.S. data reveals $12 \%$ of $2-5$ year olds, $18 \%$ of $6-11$, and $18.4 \%$ of adolescents 12-19 were observed as obese (BMI at or above the $95^{\text {th }}$ percentile). [8] It is likely that $70 \%$ of obese adolescents will grow up to be obese adults. $[9,10]$ Evidence from Brazil \& China points to a clear shift in obesity and overweight, from middle class to the poor - replicated across many countries in Asia, Africa \& Latin America. [11] The public health significance of childhood overweight and obesity is noted with the associated rates of morbidity and mortality, since elevated BMI is closely associated with morbidity and mortality. Fat tissue can have a deleterious effect on nearly every organ system, leading to: glucose intolerance, insulin resistance, type 2 diabetes, hypertension (HBP), high cholesterol, hepatic steatosis (FLD), cholelithiasis (gallstones), sleep apnea, asthma, skin conditions, menstrual abnormalities, impaired balance, orthopedic problems and metabolic syndrome. These problems lead to premature, preventable deaths. In developing countries, the burden of obesity and its complications is shifting rapidly towards the poor. Simultaneous malnutrition and overweight exist with obesity now four times more common than malnutrition in some developing countries. The rising rate of childhood overweight and obesity requires reinvestigation of the regulation of metabolism, food intake and weight gain particularly with emphasis on new environmental factors.

This public health phenomenon has increased rapidly from the 1960's. What happened in this one-generation span? A change in gene pool (not likely)? Environmental factors? Changes in the food supply? Changes in life style? Current research includes the recognition that the child inherits a gene pool, a lifestyle and a developmental trajectory from the mother. It is estimated that approximately 25 to $40 \%$ of BMI is heritable. [12] Thus, a child's weight outcome may be influenced when he/she is in utero where by lack of sleep, the intrauterine environment, and a decrease in variability of ambient temperatures, in combination with excess calories and a decrease in hard labor, may have influenced observed increases in adiposity. Being an infant of a mother with (gestational) diabetes, even if not large for gestational age, conveys an additional risk for obesity. [12-15] In addition to intrauterine factors, formula feeding with solid foods introduced before 4 months predisposes the child to a four-fold increase in obesity while breast feeding with solid foods after six months produces better outcomes. The identification of genetic determinants that partially explain the heritability of complex traits, along with the inability to define other causative genetic effects in some complex diseases, makes the new findings of epigenetic good candidates for study and manipulation.

This emerging evidence has led to the possibility of increased emphasis on pre-emptive public health interventions. New areas of research into the rise in obesity now include nutritional genomics, early exposure to excessive antibiotics changing the micro biome, epigenetic and environmental obesogens (environmental pollutants) which cause obesity by altering metabolic and homeostatic regulations, leading to weight increase. $[16,17]$ Obesogens also disrupt appetite controls, cause adipocyte or fat cell enlargement and stimulate the increase of adipocyte cell numbers.

As research advances, dietary advice based on the human genome will become more prevalent and new pharmacological interventions may be developed. Maternal "life course" and the impacts of maternal health will become a more important part of assessing etiological factors. [18] The application of nutritional epigenetic will provide new insights and understanding of the impacts of maternal health, birth outcomes, potential for quality of life and public health planning on a global scale. Many researchers view this challenge as highly important in order to avoid raising the first generation that will live a shorter time than their parents.

\section{References}

1. http://www.genome.gov/10001772

2. Gerard, S., Hayes, M., \& Rothstein, M. A. (2002). On the edge of tomorrow: fitting genomics into public health policy.J Law Med Ethics, 30(3), 173-176.

3. http://www.eaglegenomics.com/2013/08/biology-must-neverdevelop-its-own-big-data-systems/

4. Waterland, R. A., \& Jirtle, R. L. (2003). Transposable elements: targets for early nutritional effects on epigenetic gene regulation. Molecular and Cellular Biology, 23(15), 5293-5300.

5. Guarente, L., \& Kenyon, C. (2000). Genetic pathways that regulate ageing in model organisms. Nature, 408(6809), 255-262.

6. Felsenfeld, G., \& Groudine, M. (2003). Controlling the double helix. Nature, 421, 448-453.

7. Guerrero-Bosagna, C., \& Skinner, M. K. (2012). Environmentally induced epigenetic transgenerational inheritance of phenotype and disease. Molecular and Cellular Endocrinology, 354(1-2), 3-8.

8. Ogden, C. L., Carroll, M. D., Kit, B. K., \& Flegal, K. M. (2012). Prevalence of obesity and trends in body mass index among US children and adolescents, 1999-2010. JAMA: The Journal of the American Medical Association, 307(5), 483-490.

9. Dehghan, M., Akhtar-Danesh, N., \& Merchant, A. T. (2005). Childhood obesity, prevalence and prevention. Nutrition Journal, 4, 24.

10.http://www.cdc.gov/brfss/index.htm

11.http://www.yaleruddcenter.org/resources/upload/docs / seminar/2009-spring/Slides/Popkin.pdf

12. Huang, J. S., Lee, T. A., \& Lu, M. C. (2007). Prenatal programming of childhood overweight and obesity. Maternal and Child Health Journal, 11(5), 461-473. 
13. Reilly, \& John, J. (2007). Childhood obesity: an overview. Children \& Society, 21(5), 390-396.

14. Anderson, P. M., \& Butcher, K. E. (2006). Childhood obesity: trends and potential causes. The Future of children, 16(1), 19-45.

15. Apovian, C. M. (2010). The causes, prevalence, and treatment of obesity revisited in 2009: what have we learned so far? The American Journal of Clinical Nutrition, 91(1), 277S-279S.
16. Gruna, F., \& Blumberg, B. (2009). Endocrine disrupters as obesogens. Molecular and Cellular Endocrinology, 304(1-2), 19-29.

17. Del Giudice, M. (2012). Fetal programming by maternal stress: Insights from a conflict perspective. Psychoneuroendocrinology, 37(10), 161429.

18.http://mchb.hrsa.gov/lifecourse/rethinkingmchlifecourse.pdf 